\title{
PREDICCIÓN DEL TIEMPO EN LA PRUEBA DE 300 METROS EN PATINADORES JUVENILES DE RENDIMIENTO
}

\section{TIME PREDICTION IN THE 300 METER TEST OF YOUNG PERFORMANCE SKATERS}

\author{
Yennys González ${ }^{1}$, Darío Mendoza² \\ ${ }^{1}$ Doctora en Ciencias de la Actividad Física y el Deporte. Universidad Santo Tomás de Aquino, Campus San Alberto Magno, \\ Autopista Norte Avenida Los Arrayanes km 1,6, Bogotá, Colombia, e-mail: yennysgonzalez@usantotomas.edu.co; ${ }^{2}$ Magíster \\ en Epidemiología. Universidad Santo Tomás de Aquino, Campus San Alberto Magno, Autopista Norte Avenida Los Arrayanes \\ km 1,6, Bogotá, Colombia, e-mail: dariomendoza@usantotomas.edu.co
}

Rev. U..D.C.A Act. \& Div. Cient. 20(2): 247-253, Julio Diciembre, 2017

\section{RESUMEN}

El patinaje de velocidad es una disciplina competitiva de popularidad creciente; sin embargo, la evidencia científica disponible sobre regímenes de entrenamiento, de evaluación y de control es aún limitada. Por la naturaleza de la disciplina es frecuente el uso de pruebas de fuerza explosiva, para la valoración de los deportistas. El objetivo del presente estudio es predecir el rendimiento en la prueba de $300 \mathrm{~m}$, en patinadores de velocidad de categoría juvenil pista, de Bogotá, Colombia. Se evaluaron 50 patinadores (37 mujeres y 13 hombres), de la categoría juvenil. Se realizaron carreras de $300 \mathrm{~m}$, en la pista de un patinódromo y el tiempo fue cronometrado mediante un sistema de fotoceldas (Microgate Polifemo). Se determinó la altura de los saltos squat (SJ) y contra-movimiento (CMJ), con un sistema óptico de medición (OptoGait, Microgate). Se realizó una prueba de Wingate de 30s, utilizando un ciclo-ergómetro (Cyclus 2, RBM Elektronik-Automation). Se hicieron análisis bivariados y multivariados, utilizando el tiempo de la prueba de $300 \mathrm{~m}$, como variable dependiente. El análisis multivariado arrojó que la potencia media relativa $(b=-1,163$; se $b=0,326)$ y el salto desde sentadilla $(b=-1,137$; se $b=0,053)$ fueron las variables significativas $(p<0,01)$, para predecir el tiempo en la prueba de $300 \mathrm{~m}\left(\mathrm{r}^{2}=0,7\right)$. El salto squat y la potencia media relativa han mostrado ser pruebas predictoras del rendimiento en el tiempo de la prueba de $300 \mathrm{~m}$, en patinadores juveniles de Bogotá, Colombia.

Palabras clave: Potencia anaerobia, patinadores, juveniles y rendimiento.

\section{SUMMARY}

Skating race is a competitive discipline of growing popularity, however, the available scientific evidence on training, evaluation and control systems is still limited. By the nature of the discipline, using explosive force tests for the evaluation of athletes is frequent. The objective of the present study is to predict performance in the $300 \mathrm{~m}$ test of junior category speed skaters. 50 skaters ( 37 women and 13 men) from junior category were evaluated. $300 \mathrm{~m}$ races were held on the track of a skating rink, and time was measured by a photocells system (Microgate Polyphemus). Height in squat and countermovement jumps were determined using an optical system (OptoGait, Microgate). $30 \mathrm{~s}$ Wingate test was performed in a cycle-ergometer (Cyclus 2-RBM Elektronik Automation). Bivariate and multivariate analyzes were performed setting $300 \mathrm{~m}$ race time as a dependent variable. Multivariate analysis showed that average relative power $(b=$ -1.163; se $b=0.326)$ and squat jump height $(b=-1.137$; se $b=0.053)$ were the significant variables $(p<0.01)$ to predict the $300 \mathrm{~m}$ test $\left(r^{2}=0.7\right)$. The squat jump height and average relative power tests have shown to be predictive of performance in $300 \mathrm{~m}$ test in junior skaters.

Key words: Anaerobic power, skaters, juniors and performance.

\section{INTRODUCCIÓN}

El patinaje es un deporte que ha cobrado importancia en materia de investigación, gracias a su práctica en diversos contextos, tales como juegos panamericanos, campeonatos mundiales y también en prácticas recreativas y formativas (Piucco et al. 2014). Dentro de la gama de pruebas que 
posee el patinaje de carreras, sobresalen las pruebas de velocidad, que se realizan en pista, sobre una distancia de 300m, considerándose una de las pruebas que genera mayor entusiasmo entre competidores y espectadores.

Los patinadores requieren velocidad que, junto con la fuerza, constituyen la "fuerza explosiva", la cual, es una de las capacidades determinantes para el rendimiento (Hillis \& Holman, 2014). Durante la niñez, se va presentando en los practicantes de patinaje una mejora en la velocidad, aunque es en la adolescencia donde se incrementa la fuerza explosiva, gracias al desarrollo de la fuerza (Hillis \& Holman, 2014). Por lo anterior, al ser estas variables relevantes para el desempeño en el deporte, su valoración objetiva, válida y confiable en este deporte es necesaria para optimizar los recursos que dispone el entrenador, para con sus deportistas (Piucco et al. 2015).

Gestos técnicos de otras disciplinas, como el ciclismo, combinado con ejercicios específicos para patinadores han sido efectivos, para la mejora de las capacidades aerobias y anaerobias, en patinadores élite (Stangier et al. 2016). Ahora, en la evaluación de patinadores, por lo general, se han tomado pruebas ya validadas para ciclistas o corredores, pero algunos patrones de movimiento, especialmente realizados por patinadores de pista, paso cruzado e inclinación en las curvas, postura, entre otros, hacen necesario el estudio sobre la valoración específica en esta disciplina (Stangier et al. 2016).

Los patinadores colombianos, se destacan en este tipo de pruebas, tanto de velocidad como de fondo y obtienen registros mundiales, incluyendo, actualmente, el récord en la rama masculina, que se encuentra en 23,41 s, en la prueba de 300m pista (Record - FIRS - Fédération Internationale Roller Sports, 2016). Debido a este caso y otros resultados destacados que han obtenido los patinadores de este país, existe interés por estudiar variables relacionadas con el rendimiento, en este tipo de disciplinas.

En otros deportes, como natación, ciclismo, atletismo de carrera y triatlón, se ha estudiado la asociación de diversos componentes antropométricos, de entrenamiento y variables relacionadas con experiencias previas. Otros estudios, se centran más en la relación de componentes antropométricos y biomecánicos con el rendimiento, pero se han aplicado a deportistas de patinaje de velocidad sobre hielo (Konings et al. 2015; Haug et al. 2015). En el patinaje de carreras, se encuentran pocos estudios que predicen el rendimiento y se centran más en pruebas de larga duración (Knechtle et al. 2011; Lozada, 2015). Por tal motivo, el objetivo del presente estudio es el de predecir, a partir de características de valoración de la capacidad anaerobia, por medio de pruebas de saltos SJ y CMJ y variables de ciclismo anaerobio (Wingate), el tiempo en la prueba de 300m pista, en patinadores juveniles de Bogotá, Colombia.

\section{MATERIALES Y MÉTODOS}

Participantes del estudio. Un total de 50 patinadores juveniles inscritos a un club de la liga de Bogotá de patinaje, con licencia federativa en vigor departamental, Colombia (2.640msnm) participaron voluntariamente en este estudio. El consentimiento informado fue firmado y autorizado por los padres de familia, a quienes se les informó, por escrito, acerca de las características, los objetivos y los riesgos de este estudio. Los patinadores, en su mayoría, son menores de edad, promedio 15,14 años ( $\pm 1,88$ años), promedio de peso y talla, 52,16kg ( $\pm 8,37 \mathrm{~kg})$ y $1,5 \mathrm{~m}( \pm 0,1 \mathrm{~m})$, respectivamente. Dichos patinadores entrenaban una media de 20 horas semanales. El estudio fue aprobado por el comité de investigaciones institucional de la Universidad Santo Tomás y se cumplieron los principios éticos, contemplados en la Declaración de Helsinki y la Resolución 8430 de 1993 del Ministerio de Salud de Colombia.

Pruebas físicas. La toma de pruebas físicas, se llevó a cabo durante el periodo precompetitivo general, en el 2015, los sábados, entre las 8:00 y 10:00 a.m., en las instalaciones de un patinódromo reglamentario, ubicado en un parque recreo deportivo, de Bogotá. La prueba de 300m, se realizó en la pista del patinódromo, previo calentamiento general y especial de 20 minutos, orientado por el entrenador del grupo. Se realizaron tres intentos, con un intervalo de tres minutos entre repeticiones y se registró el mejor tiempo de ellos. Todos los intentos fueron cronometrados, mediante un sistema de fotocélulas de luz infrarroja tipo reflejo, para la medición de tiempos con precisión, de $100 \mathrm{~Hz}$, modelo WL34-R240, Marca Sick ${ }^{\circledR}$, Potencia de encendido: Relevo, SPDT. Aislado. Max. Frecuencia de encendido: 10/s. Tiempo de demora: tabla, ajustable 0.5 a 10 s. (Alemania).

Con ocho días de diferencia, se llevaron a cabo las pruebas de fuerza explosiva. Inicialmente, y con el fin de potencializar el rendimiento, se realizó calentamiento, consistente en estiramientos activos y saltos previos (Turki et al. 2011). A continuación, se ingresaron los datos de cada participante en el software, de un sistema óptico de medición, para la fuerza explosiva (OptoGait, Microgate Italia, Bolzano-Bozen, Italia), con precisión temporal de 1 milisegundo y se procedió a realizar la prueba del SJ y, posteriormente, la prueba de CMJ. Este dispositivo ha demostrado, previamente, una fuerte validez, concurrente con plataformas de fuerza, así como también una excelente confiabilidad test-re test, para la estimación de la altura en saltos verticales (Glatthorn et al. 2011). Los patinadores ejecutaban las pruebas en grupos de ocho personas, en un coliseo cubierto, contiguo al patinódromo y acondicionado para que todas las mediciones 
se realizaran bajo las mismas condiciones. El tiempo de recuperación entre las repeticiones de los saltos fue de tres minutos, en los que practicaban estiramientos activos. La mejor altura del salto fue tenida en cuenta, para el análisis en las dos pruebas.

La prueba de Wingate, se realizó sobre un ciclo-ergómetro (Cyclus 2, RBM Elektronik-Automation $\mathrm{GmbH}$, Leipzig, Alemania), la potencia con un error de $2 \%$ (para valores menores de 100 vatios, máximo 2 vatios), la cadencia con un error maximal \pm 1 RPM y la retroalimentación mecánica de HBM T5 torque, utilizado en otros estudios (Tinwala et al. 2017). La mayoría de los participantes, llevó su propia bicicleta y, en el caso de los que no disponían de una, se acondicionó un marco de bicicleta de otro participante, para que se ajustara a las medidas corporales. El calentamiento consistió en 10 minutos a una cadencia de 60 rpm, con una carga de 30 vatios; posteriormente, se empezó la prueba y los participantes fueron animados verbalmente a realizar su mejor esfuerzo, por un tiempo de 30 segundos. Se tuvieron en cuenta las variables de potencia máxima anaerobia y potencia media, en términos absolutos y relativos al peso corporal, para el análisis.

Análisis estadístico. La distribución de los datos, se determinó mediante la prueba de Kolmogorov-Smirnov, encontrándose que todas las variables se ajustan a una curva normal $(p>0,05)$. Los datos son presentados como promedios y desviaciones estándar para todas las variables analizadas. Se realizó análisis de regresión de "Pearson" con el tiempo de la prueba de $300 \mathrm{~m}$, para identificar las variables que serían tenidas en cuenta en un modelo de regresión múltiple (por pasos). Fue empleado un nivel de significancia de 0,05 y se empleó el software IBMSPSS versión 23, para el tratamiento de la información (Licencia, Universidad Santo Tomás).

\section{RESULTADOS Y DISCUSIÓN}

Un total de 50 jóvenes patinadores participaron en el estudio voluntariamente, 37 mujeres (74\%) y 13 hombres (26\%). La tabla 1 presenta las características de los participantes y los resultados promedio en las pruebas físicas, así como los coeficientes de correlación bivariada de "Pearson" con respecto al tiempo de la prueba de velocidad de 300m. Los resultados en la prueba de $300 \mathrm{~m}$ fueron: promedio general 29,67s ( $\pm 2,23$ ) de duración; en mujeres, 30,42 s ( $\pm 2,04$ s) y $27,55 \mathrm{~s}( \pm 1,07 \mathrm{~s})$.

La edad, la talla, los saltos SJ y en CMJ, la potencia máxima y la media a partir de la prueba de Wingate estuvieron significativamente correlacionadas con el tiempo de carrera de la prueba de $300 \mathrm{~m}$. Un análisis de regresión múltiple paso a paso mostró que las variables de potencia media relativa (Figura 1) y salto desde sentadilla (Figura 2) fueron las variables que obtuvieron valores de correlación más altos con el tiempo de carrera.

Tabla 1. Características y pruebas físicas de los participantes con respecto al tiempo de carrera de velocidad de $300 \mathrm{~m}$ ( $\mathrm{n}=$ 50).

\begin{tabular}{|l|c|c|c|c|}
\hline \multicolumn{1}{|c|}{ Variable } & Promedio & D.E.* & R & Valor $\mathbf{p}$ \\
\hline Edad (años) & 15,14 & 1,885 & $-0,457$ & 0,001 \\
\hline Peso $(\mathrm{kg})$ & 52,1600 & 8,37910 & $-0,272$ & 0,056 \\
\hline Talla $(\mathrm{m})$ & 1,5910 &, 10170 & $-0,319$ & 0,024 \\
\hline IMC $\left(\mathrm{kg} / \mathrm{m}^{2}\right)$ & 20,5547 & 2,25669 & $-0,009$ & 0,95 \\
\hline Salto en contra movimiento CMJ (cm) & 29,8560 & 6,74471 & $-0,79$ & $<0,001$ \\
\hline Salto squat SJ (cm) & 27,6660 & 6,15552 & $-0,78$ & $<0,001$ \\
\hline Potencia máxima (vatios) & 530,3420 & 168,56026 & $-0,523$ & $<0,001$ \\
\hline Potencia media (vatios) & 416,4460 & 88,17957 & $-0,652$ & $<0,001$ \\
\hline Potencia máxima relativa (vatios/kg) & 10,2480 & 2,29794 & $-0,487$ & $<0,001$ \\
\hline Potencia media relativa (vatios/kg) & 8,0480 &, 90919 & $-0,801$ & $<0,001$ \\
\hline
\end{tabular}

*D.E.: Desviación Estándar. 


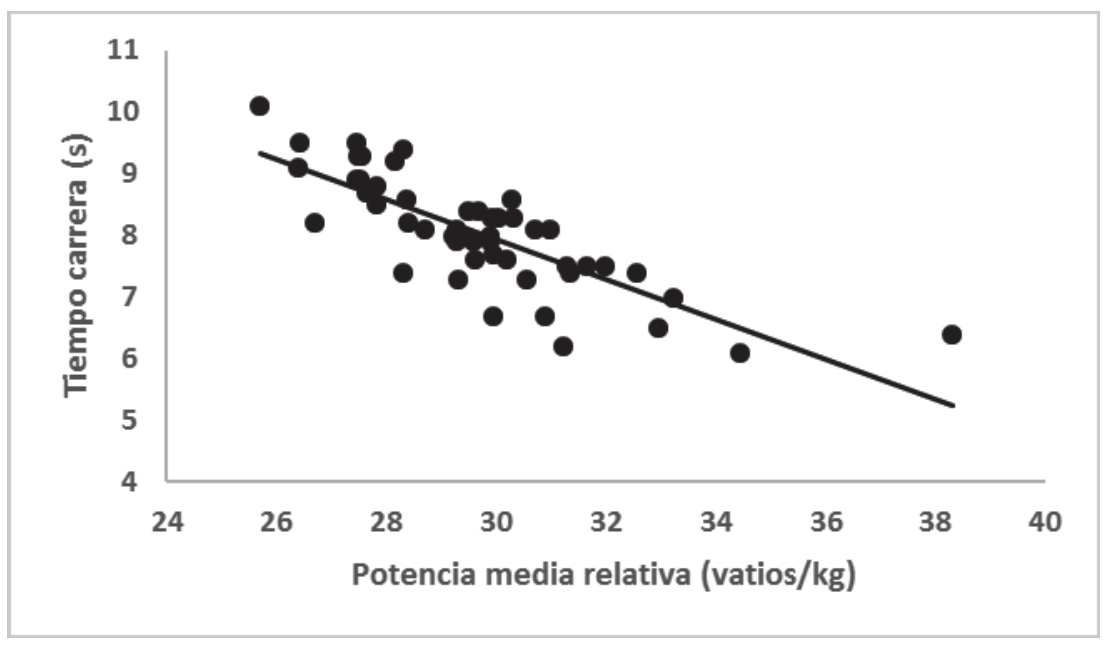

Figura 1. Potencia anaerobia media relativa fue significativamente e inversamente relacionada con el tiempo de carrera de $300 m(n=50)(r=-0,8 ; p<0,001)$.

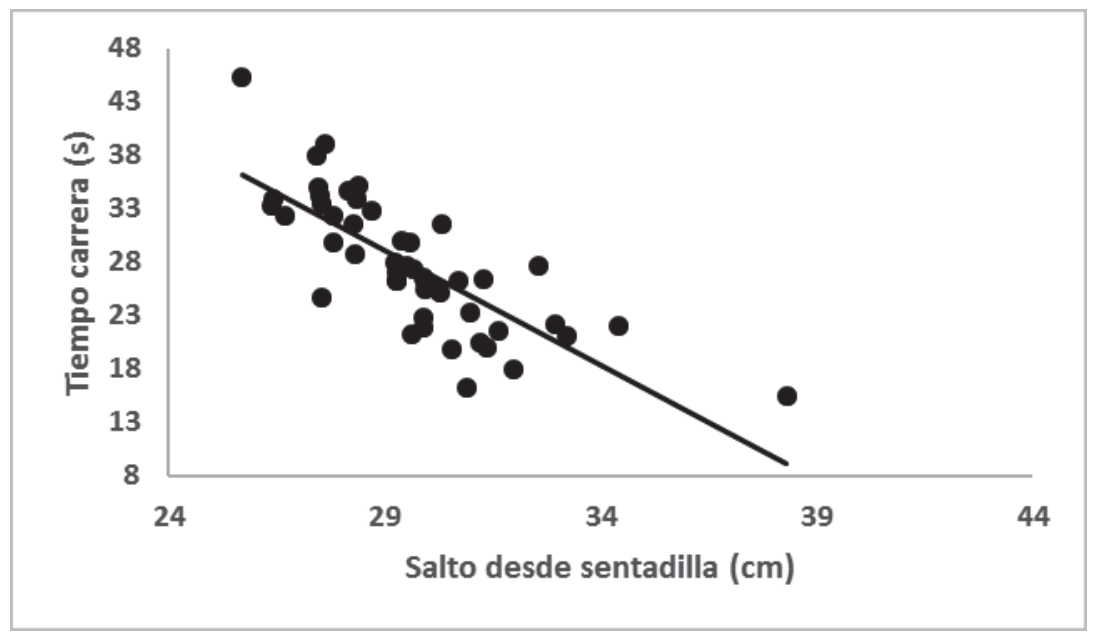

Figura 2. El SJ o Salto desde sentadilla fue significativamente e inversamente relacionado con el tiempo de carrera de $300 \mathrm{~m}$ $(n=50(r=-0,78 ; p<0,001)$.

A partir del análisis multivariado, se pudo determinar la existencia de una relación entre las variables que viene explicada por la ecuación:

$$
Y=43,433-1,163 X_{1}-0,137 X_{2}
$$

Donde: $Y$ es el tiempo en la prueba de $300 \mathrm{~m}: X_{1}$, la potencia anaeróbica media relativa y $X_{2}$, el SJ. En este modelo, el coeficiente de determinación fue de 0,70 y el error cuadrático medio fue 1,44 . En la tabla 2, se muestran los coeficientes tipificados y sus valores de probabilidad.
Se evaluó la independencia de los errores por medio del estadístico de Durbin-Watson, siendo de un valor de 2,11, por lo que se presupone este supuesto en el modelo. El factor de inflación de la varianza (FIV) fue cercano a 1 en todas las variables, por lo que se presume la no multicolinealidad entre las variables (Field, 2013).

El patinaje de velocidad es una disciplina competitiva de popularidad creciente (Bohórquez et al. 2016); sin embargo, la evidencia científica disponible sobre regímenes de entrenamiento, de evaluación y de control es aún limitada. Adicionalmente, la práctica formativa y recreativa es cada vez más frecuente, llamando la atención sobre la necesidad 
Tabla 2. Coeficiente B para tiempo en la prueba de velocidad de $300 \mathrm{~m}$ como variable dependiente $(n=50)$.

\begin{tabular}{|l|c|c|c|}
\hline Variable & B & se b & b estandarizado \\
\hline Constante & 43,433 & 1,726 & \\
\hline Edad & $-0,005$ & 0,119 & $-0,004$ \\
\hline Sexo & $-0,272$ & 0,529 & $-0,054$ \\
\hline Potencia media relativa & $-1,163$ & 0,326 & $-0,474^{*}$ \\
\hline Salto desde sentadilla & $-0,137$ & 0,053 & $-0,379^{*}$ \\
\hline
\end{tabular}

* El valor de los coeficientes es significativo $(p<0,01)$.

de realizar estudios también en las categorías infantil y juvenil (Piucco et al. 2014). Por la naturaleza de la disciplina es frecuente el uso de pruebas de fuerza explosiva para la valoración de los deportistas. Tanto las pruebas de salto como de ciclismo anaerobio son mecanismos de valoración ampliamente utilizados por los entrenadores, para controlar el progreso de los deportistas y este estudio permitió identificar los indicadores que más se relacionaron con el rendimiento en patinadores juveniles de Bogotá, Colombia. Se pudo determinar, que la altura del SJ y la potencia máxima relativa (prueba de Wingate) permiten predecir el tiempo en la prueba de velocidad de $300 \mathrm{~m}$, en patinadores juveniles.

Nuestro estudio, realizado con 50 patinadores juveniles, obtuvo tiempos promedio en la prueba de $300 \mathrm{~m}$ de 30,42 s, en mujeres y 27,55 s, en hombres. Para propósitos comparativos, 6 patinadores de Canadá y 3 de Holanda, hombres bien entrenados, con una estrategia de paso rápido, registraron tiempos sobre la misma distancia de $26,18 \mathrm{~s}$ ( \pm 0,79s); sin embargo, estos participantes son de categoría mayores y han participado en campeonatos regionales y nacionales en sus países, lo que explica el mejor desempeño, con respecto a los juveniles de Bogotá (Stoter et al. 2016).

La utilidad de la potencia anaeróbica, para predecir el desempeño en pruebas de velocidad, ha sido previamente documentada, en jugadores de Hockey sobre hielo (Potteiger et al. 2010; Peyer et al. 2011). En el estudio de Potteiger, se analizó la correlación entre el tiempo registrado en 6 carreras de $89 \mathrm{~m}$ sobre pista de hielo y se encontró correlación significativa con la potencia pico relativa, medida mediante prueba de Wingate, de 30s. En contraste, nosotros encontramos que, dentro de todas las variables determinadas, mediante esta misma prueba, fue la potencia media relativa (por kilogramo de masa corporal) la que mostró ser predictora del tiempo, en la prueba de $300 \mathrm{~m}$. Estos resultados, se pueden explicar, porque en el mencionado estudio, las pruebas de $89 \mathrm{~m}$ sobre hielo, se caracterizan por una mayor velocidad, por lo que se puede asumir que son esfuerzos predominantemente anaeróbicos alácticos, lo que se refleja mejor en el valor de potencia máxima, mientras que, en nuestro estudio, por tratarse de una prueba de $300 \mathrm{~m}$ sobre pista de asfalto, podemos asumir que se trata de un esfuerzo predominantemente anaeróbico láctico, lo que no se refleja, tanto en la potencia pico como sí en la potencia promedio, durante toda la prueba de Wingate, de 30 s.

En investigaciones pasadas, se ha demostrado que, patrones de movimiento, como el ciclismo o la carrera, son igualmente efectivas para mejorar el rendimiento de velocidad y de resistencia aerobia, en practicantes de patinaje (Stangier et al. 2016). Por estos motivos, no es raro encontrar valoraciones sobre cicloergómetro o banda rodante aplicadas a patinadores de distintos niveles y edades; sin embargo, en casos de duda o necesidad de una mayor precisión, se recomienda la aplicación de pruebas específicas (Stangier et al. 2016).

Aunque la altura de salto es un indicador ampliamente aceptado de fuerza explosiva, se ha observado que el desempeño obtenido y la estrategia utilizada para ejecutar los diferentes tipos de salto son específicos, de acuerdo con el tipo de entrenamiento (Arabatzi \& Kellis, 2012). Así quedó en evidencia tras observarse mediante análisis cinético y cinemático, que la ejecución del SJ es significativamente diferente entre diversas modalidades de entrenamiento de fuerza explosiva: levantadores de peso, en comparación con patinadores de velocidad; tales diferencias no se observaron en el CMJ (Haug et al. 2015). En nuestros resultados, aunque inicialmente en el análisis bivariado se observó correlación de la altura de salto, tanto el ejecutado SJ como el de CMJ, con respecto al tiempo en la prueba de velocidad, al realizar el análisis multivariado solo el primero mostró ser predictor del tiempo en los $300 \mathrm{~m}$. Esto se puede explicar porque, a diferencia del CMJ, el SJ se asemeja más al gesto natural de patinaje, que típicamente es con el cuerpo inclinado hacia la pista, con rodillas y codos flexionados, en posición fija.

Por otra parte, las variables evaluadas relacionadas con parámetros antropométricos, como la talla y el peso, no mostraron relación alguna con el rendimiento en la 
carrera de $300 \mathrm{~m}$, debido, principalmente, a que todos los participantes se encontraban en condiciones normales para estos parámetros, teniendo en cuenta que la muestra evaluada se encuentra en proceso de entrenamiento para altos logros; estos resultados son consistentes con los encontrados en previas investigaciones (Konings et al. 2015). Por el contrario, un estudio llevado a cabo con practicantes de patinaje recreativos (Knechtle et al. 2011) mostro correlaciones positivas significativas con edad $(r=0,3)$, masa corporal $(r=0,42)$ e índice de masa corporal $(0,35)$, los cuales, son opuestos a los resultados del presente estudio en edad $(r=-0,45)$ y el IMC, que no presentó significancia. Las razones de esta diferencia radican en el promedio de edad de los estudios (40,7 versus 15,7 años, en nuestro estudio), el nivel de los participantes, que era recreativo en ese estudio, mientras que fue competitivo en la presente investigación y, finalmente, el tipo de prueba: $111 \mathrm{~km}$ frente a $300 \mathrm{~m}$. Aunque, sin lugar a duda, los estudios son diferentes en su variable dependiente, resulta ilustrativa la comparación entre las variables predictoras de desempeño en pruebas de resistencia aerobia y de velocidad en el mismo deporte. En este sentido, llama la atención que, mientras en ese mismo estudio se encontró correlación positiva con porcentaje de grasa (adipometría), en otro, Potteiger et al. (2010) informaron una correlación negativa entre el porcentaje de grasa (pletismografía por desplazamiento de aire) y el tiempo en una carrera de $89 \mathrm{~m}$, en jugadores de hockey sobre hielo, con promedio de edad de 20 años. A partir de lo anterior, se puede plantear la necesidad de estudiar la utilidad de variables antropométricas y de composición corporal, tales como perímetros de pierna, porcentaje de grasa y masa muscular, como determinantes o predictores de desempeño en pruebas de velocidad, específicamente.

El patrón de movimiento de los patinadores es caracterizado por una postura de rodillas, de caderas y de codos, flexionados en ángulo fijo, con el tronco inclinado, para favorecer la técnica y la aerodinámica (Stoter et al. 2016). Por tanto, es lógico que, a partir de la prueba de fuerza explosiva del SJ pueda predecir el rendimiento en pruebas de velocidad de este deporte. Asimismo, la potencia media relativa, obtenida a partir de la prueba de cicloergómetro, a pesar de no ser un patrón de movimiento específico, sí impone una demanda energética que es similar a la prueba de velocidad de $300 \mathrm{~m}$, en cuanto a potencia energética y predominancia en el tiempo. Estas pruebas han sido las variables predictoras del tiempo en la prueba de velocidad en jóvenes patinadores de Bogotá, lo que puede contribuir a los procesos de identificación, de búsqueda y de desarrollo de los talentos deportivos (Vaeyens et al. 2008), en esta disciplina.

Conflictos de intereses. El manuscrito fue preparado y revisado con la participación de todos los autores, quienes declaramos que no existe conflicto de intereses que ponga en riesgo la validez de los resultados presentados. Financiación: Este estudio fue financiado por el Fondo para el desarrollo de proyectos de Investigación de la Universidad Santo Tomás de Aquino, sede Bogotá.

\section{BIBLIOGRAFÍA}

1. ARABATZI, F.; KELLIS, E. 2012. Olympic Weightlifting Training Causes Different Knee Muscle-Coactivation Adaptations Compared with Traditional Weight Training. The Journal of Strength \& Conditioning Research, 26(8):2192-2201.

2. BOHÓRQUEZ-PÁEZ, D.A.; ROJAS-RUÍZ, F.J.; GIMÉNEZ-FUENTES-GUERRA, F.J. 2016. Eficiencia de las salidas frontal y lateral para la prueba de pista 300 metros CRI, patinaje de velocidad sobre ruedas. Orinoquia. 20(1):77-83.

3. FIELD, A. 2013. Discovering Statistics Using IBM SPSS Statistics. SAGE; 953p.

4. GLATTHORN, J.F.; GOUGE, S.; NUSSBAUMER, S.; STAUFFACHER, S.; IMPELLIZZERI, F.M.; MAFFIULETTI, N.A. 2011. Validity and reliability of Optojump photoelectric cells for estimating vertical jump height. J. Strength Cond. Res. 25:556-560.

5. HAUG, W.B.; DRINKWATER, E.J.; MITCHELL, L.J.; CHAPMAN, D.W. 2015. The relationship between start performance and race outcome in elite 500-m short-track speed skating. Int. J. Sports Physiology and Performance. 10(7):902-906.

6. HAUG, W.B.; SPRATFORD, W.; WILLIAMS, K.J.; CHAPMAN, D.W.;-DRINKWATER, E.J. 2015. Differences in End Range of Motion Vertical Jump Kinetic and Kinematic Strategies Between Trained Weightlifters and Elite Short Track Speed Skaters. J. Strength and Conditioning Res. / National Strength \& Conditioning Association, 29(9):2488-2496.

7. HILLIS, T.L.; HOLMAN, S. 2014. The Relationship between Speed and Technical Development in Young Speed Skaters. Int. J. Sports Science \& Coaching. 9(2):393-400.

8. KNECHTLE, B.; KNECHTLE, P.; RÜST, C.A.; SENN, O.; ROSEMANN, T.; LEPERS, R. 2011. Predictor variables of performance in recreational male long-distance inline skaters. J. Sports Sciences, 29(9):959966. 
9. KONINGS, M.J.; ELFERINK-GEMSER, M.T.; STOTER, I.K.; VAN DER MEER, D.; OTTEN, E.; HETTINGA, F.J. 2015. Performance characteristics of long-track speed skaters: a literature review. Sports Medicine (Auckland, N.Z.). 45(4):505-516.

10. LOZADA, J.L. 2015. Comparación de las características antropométricas entre patinadores de velocidad medallistas y no medallistas. Actividad Física y Ciencias. $7(1): 1-15$.

11. PEYER, K.L.; PIVARNIK, J.M.; EISENMANN, J.C.; VORKAPICH, M. 2011. Physiological characteristics of National Collegiate Athletic Association Division I ice hockey players and their relation to game performance. The J. Strength \& Conditioning Res. 25(5):1183-1192.

12. PIUCCO, T.; DOS SANTOS, S.; DANTAS DE LUCAS, R.; ACHE-DIAS, J. 2015. Nuevo test incremental para patinadores velocistas sobre una superficie plana deslizante: análisis de fiabilidad y comparación con un test de ciclismo. Apunts Medicina de l"Esport. 50(186):57-63.

13. PIUCCO, T.; DOS SANTOS, S.G.; DE LUCAS, R.D. 2014. Patinação de velocidade in-line: uma revisão sistemática. Rev. Andaluza de Medicina del Deporte. 7(4):162-169.

14. POTTEIGER, J.A.; SMITH, D.L.; MAIER, M.L.; FOSTER, T.S. 2010. Relationship between body composition, leg strength, anaerobic power, and on-ice skating performance in division I men's hockey athletes. Journal of Strength and Conditioning Research / National Strength \& Conditioning Association. 24(7):1755-1762.

15. Record - FIRS - Fédération Internationale Roller Sports. 2016. Disponible desde Internet en: http://www.rollersports.org/discipline/speed/record (con acceso 08/03/17).

16. STANGIER, C.; ABEL, T.; HESSE, C.; CLAEN, S.; MIERAU, J.; HOLLMANN, W.; STRÜDER, H.K. 2016. Effects of Cycling vs. Running Training on Enduran- ce Performance in Preparation for Inline Speed Skating. Journal of Strength and Conditioning Research / National Strength \& Conditioning Association. 30(6):1597-1606.

17. STANGIER, C.; ABEL, T.; MIERAU, J.; GUTMANN, B.; HOLLMANN, W.; STRUDER, H.K. 2016. Comparison of sport-specific and non-specific exercise testing in inline speed skating. The Journal of Sports Medicine and Physical Fitness. 56(4):406-414.

18. STANGIER, C.; ABEL, T.; MIERAU, J.; HOLLMANN, W.; STRÜDER, H.K. 2016. Effects of Cycling Versus Running Training on Sprint and Endurance Capacity in Inline Speed Skating. J. Sports Science \& Medicine. 15(1):41-49.

19. STOTER, I.K.; MACINTOSH, B.R.; FLETCHER, J.R.; POOTZ, S.; ZIJDEWIND, I.; HETTINGA, F.J. 2016. Pacing Strategy, Muscle Fatigue, and Technique in 1500-m Speed-Skating and Cycling Time Trials. Int. J. Sports Physiology and Performance. 11(3):337343.

20. TINWALA, F.; CRONIN, J.; HAEMMERLE, E.; ROSS, A. 2017. Eccentric Strength Training: A Review of the Available Technology. Strength Cond. J. 39(1):3247.

21. TURKI, O.; CHAOUACHI, A.; DRINKWATER, E.J.; CHTARA, M.; CHAMARI, K.; AMRI, M.; BEHM, D.G. 2011. Ten minutes of dynamic stretching is sufficient to potentiate vertical jump performance characteristics. Journal of Strength and Conditioning Research / National Strength \& Conditioning Association. 25(9):2453-2463.

22. VAEYENS, R.; LENOIR, M.; WILLIAMS, A.M.; PHILIPPAERTS, R.M. 2008. Talent identification and development programmes in sport: current models and future directions. Sports Medicine (Auckland, N.Z.). 38(9):703-714.

Recibido: Junio 22 de 2017

Aceptado: Octubre 10 de 2017

Cómo citar:

González, Y.; Mendoza, D. 2017. Predicción del tiempo en la prueba de 300 metros en patinadores juveniles de rendimiento. Rev. U.D.C.A Act. \& Div. Cient. 20(2): 247-253. 